\title{
The Need for Transfer Learning in CRISPR-Cas Off-Target Scoring
}

\author{
Pavan K. Kota ${ }^{\dagger 1}$, Yidan Pan ${ }^{\dagger 1}$, Hoang-Anh Vu ${ }^{1}$, Mingming Cao ${ }^{1}$, Richard G. Baraniuk ${ }^{2}$, \\ and Gang Bao ${ }^{1}$ \\ ${ }^{1}$ Department of Bioengineering, Rice University \\ ${ }^{2}$ Department of Electrical and Computer Engineering, Rice University \\ ${ }^{\dagger}$ These authors contributed equally to the paper.
}

August 28, 2021

\begin{abstract}
Motivation: The scalable design of safe guide RNA sequences for CRISPR gene editing depends on the computational "scoring" of DNA locations that may be edited. As there is no widely accepted benchmark dataset to compare scoring models, we present a curated "TrueOT" dataset that contains thoroughly validated datapoints to best reflect the properties of in vivo editing. Many existing models are trained on data from high throughput assays. We hypothesize that such models may suboptimally transfer to the low throughput data in TrueOT due to fundamental biological differences between proxy assays and in vivo behavior. We developed new Siamese convolutional neural networks, trained them on a proxy dataset, and compared their performance against existing models on TrueOT.

Results: Our simplest model with a single convolutional and pooling layer surprisingly exhibits state-ofthe-art performance on TrueOT. Adding subsequent layers improves performance on the proxy dataset while compromising performance on TrueOT. We demonstrate that model complexity can only improve performance on TrueOT if transfer learning techniques are employed. These results suggest an urgent need for the CRISPR community to agree upon a benchmark dataset such as TrueOT and highlight that various sources of CRISPR data cannot be assumed to be equivalent.

Availability and Implementation: Our code base and datasets are available on GitHub at github.com/baolab-rice/CRISPR_OT_scoring.
\end{abstract}

\section{Introduction}

CRISPR-Cas9 systems are engineered for site- and sequence-specific genome editing [1,2]. The S. pyogenes Cas9 (SpCas9) system is the most common variant and typically requires a 20-base guide RNA (gRNA) that targets a DNA sequence upstream of a Protospacer Adjacent Motif (PAM) sequence of "NGG" 3.4]. The relatively high efficiency of SpCas9 systems and ease of construction in performing gene editing have led to a revolution in life sciences and medicine. However, unintended editing at "off-target" (OT) DNA sites is a major concern for gene editing applications [3. The hybridization of the gRNA to target DNA tolerates imperfect sequence homology which causes OT activity [5 7. The resulting double strand breaks (DSBs) can induce undesired mutations that vary gene expression levels or even disrupt genes, and multiple DSBs can result in chromosomal rearrangement or severe DNA damage [8, 9]. Therefore, rational designs of gRNAs with minimal OT activity are critical for both scientific studies and safe therapeutic applications of CRISPR-Cas9 systems.

As experimentally screening target DNA sites for potential OT activity is tedious and expensive, computational techniques are critical to scalably evaluate gRNA designs [10. This evaluation has three phases for a given gRNA: screening for potential OTs across the whole genome, scoring the list of targets, and aggregating the scores into an overall gRNA quality metric [11]. In this work, we focus on scoring. Scoring models initially used hypotheses on relevant sequence features that affect editing activity 12 15]. Increased 
availability of CRISPR gene-editing data has enabled machine learning (ML) to directly learn relevant features from data [16. Given a gRNA-target sequence pair as input, models predict a label that is either binary for classification (predicting whether a gRNA edits a target site) [17 19] or continuous for regression (predicting editing efficiency) [1, 20]. With few exceptions 19,20, most scoring algorithms focus only on mismatches between the gRNA and target sequences without accounting for bulges. However, CRISPR-Cas9 systems are able to generate DSBs at sites with bulges in both in vitro and in vivo settings among multiple cell types $6,21,22$.

Training a ML scoring model capable of assessing gRNA-target pairs with bulges is essential, but the available datasets are fundamentally limited. Ideally, datasets would label pairs based on the gRNA's tendency to edit the target site in vivo, but such validation is painstaking. Whole-genome sequencing is limited by the sequencing depth and is generally unable to detect OT sites with less than $5 \%$ editing efficiency 23 25]. Therefore, many ML approaches rely on datasets generated by genome-wide high throughput methods based on proxies such as the insertion rate of a double-stranded DNA tag (GUIDE-seq 26]) or the cleavage rate in vitro (CIRCLE-seq [27, CHANGE-seq 22]) and in vivo (DISCOVER-seq [28, HTGTS [29]). Unfortunately, recent studies have shown that proxy assays have low concordance among each other and with validated in vivo editing [22,30]. Perhaps due to this variable performance between assays, there is no gold standard benchmark dataset on which to compare scoring models despite the pervasive use of such benchmarks in other applications of ML. A carefully selected benchmark is urgently needed to avoid biased progressing in the field of ML applications to CRISPR 31].

Therefore, our first contribution is the curation of a novel benchmark "TrueOT" dataset. TrueOT contains 1903 binary-labeled datapoints that were thoroughly validated by mutation rates in vivo. We argue that in vivo editing prediction on gRNAs that are not seen during model training is the best performance metric for any scoring model. The use of proxy datasets to train models implicitly assumes that interactions between gRNAs and DNA are independent of the setting. Our second contribution is the unraveling of this assumption through the evaluation of a suite of Siamese convolutional neural networks. As we will discuss, this architecture is particularly helpful for testing the assumption of dataset equivalence through the lens of transfer learning. We trained these networks on a "Proxy Dataset" and found that our "S1C" model with a single large convolutional and pooling layer achieved state-of-the-art generalization to TrueOT among bulgecapable models. Only Elevation [11] and predictCRISPR [18], models that only account for mismatches and require equal-length gRNA-target pairs, performed better on the relevant subsets of TrueOT. We found that more complex Siamese models could improve performance on the Proxy Dataset while compromising generalization to TrueOT. A core principle of transfer learning with neural networks is that initial convolutional layers capture features that generalize well between datasets while deeper layers must be retuned accordingly on a portion of the target dataset 32$]$. We believe this framework explains our results.

Our core thesis is that if TrueOT is an acceptable benchmark dataset, then future efforts in scoring model development must consider applying transfer learning principles of ML to account for the underlying gap between proxy assays and in vivo editing behavior. TrueOT currently contains too few datapoints to train deep networks on directly. Still, we perform a preliminary demonstration of transfer learning through a novel dimensionality reduction on the output of our S1C to enable the tuning of a much smaller network. Our TrueOT benchmark and Siamese models serve as potent starting points for continued research into this problem. As more data is added to TrueOT, the efficacy of transfer learning will naturally improve along with the confidence in the safety of designed gRNAs.

\section{Methods}

\subsection{Dataset Curation}

\subsubsection{TrueOT}

Currently, no assay enables direct genome-wide measurement of CRISPR-induced DNA sequence alteration with high sensitivity and throughput, significantly limiting the size of TrueOT. We defined three criteria for the inclusion of experimental data in TrueOT: (1) the experiments were performed in living cells, retaining the information that is missing from in vitro settings; (2) the OT editing efficiencies were evaluated by directly measuring the target sequence mutation rate, the common standard in clinical settings; (3) the OTs 
have a chromosomal position provided by original studies or a unique chromosomal position that can be retrieved in the reference genome using COSMID 13. This filtering results in 1903 unique datapoints with 36 unique gRNAs from 11 different studies. Ten studies' datapoints were experimentally validated through next-generation sequencing of PCR amplicons $[9,22,33,40$. We positively labeled gRNA-target pairs with an editing rate greater than $0.1 \%$, a commonly accepted threshold for deeming OTs. Although some scoring models use continuously valued editing efficiency for regression, we suggest that in evaluating potential OTs, any degree of editing may be dangerous and should be flagged accordingly. One study experimentally validated datapoints by T7 Endonuclease I for which we used the original study's labels [5]. In determining which datapoints have bulges, we used the original studies' alignment information to avoid adding a source of variability (Fig. S1). Among the 280 positive OTs in TrueOT, 10 had bulges, highlighting the need for bulge-aware scoring models. For further details on the included studies, see Table S1.

In our uploaded dataset (Table S2), we decided to keep all 1903 available datapoints that were performed in unique studies or unique experimental conditions. For example, five of the gRNAs appeared in two studies 5, 35 that used different cell types which results in some datapoints in TrueOT with the same gRNAtarget pair. Notably, these datapoints do not always have the same label, reflecting commonly observed cell-type dependencies in gene editing [41. In model evaluation, we filtered for the 1841 unique triplets of $\{$ gRNA, target, label\}. By doing so, we focus on the aggregate ability of models to make predictions on sequence information alone and reduce any ambiguous gRNA-target pairs to one instance of each a positive and negative label. Table 1 includes this duplicate removal in its description of TrueOT.

\subsubsection{Proxy Dataset}

For training our models, we combined a dataset from a recent review [42] and the training set from CRISTA [20]. The former includes datapoints from several publications but lacks datapoints with bulges, motivating the inclusion of the latter. We excluded datapoints with gRNAs in TrueOT from this combined dataset, ensuring that our models' performance on TrueOT reflects their ability to make in vivo editing predictions on unseen gRNAs. After this filtering, our Proxy Dataset has 3505 remaining datapoints from proxy assays, predominantly GUIDE-SEQ and HTGTS.

\subsection{Pairwise Comparisons}

In addition to several rule-based models $6,10,13,15,43]$, we chose the following recent ML models for their high performance as evaluated by a recent review [42]: CRISTA [20], Elevation [11], predictCRISPR 18], CNN_std [17]. We also include CRISPR-NET [19, a recently developed bulge-aware scoring model. To evaluate the published models on TrueOT, we do not have the luxury of retroactively removing datapoints in the original training sets that contain gRNAs in TrueOT. Instead of retraining existing models on new datasets, we evaluated the performance of the published models on subsets of TrueOT involving gRNAs that were not used in the training of the original model. We also filtered datapoints for which the model cannot produce an output such as a bulge-containing datapoint with a mismatch-only algorithm. Table 1 clarifies this process for the ML baselines. Most rule-based techniques were restricted to the 1614 datapoints without DNA-RNA bulges except COSMID. which can tolerate up to one bulge, allowing its evaluation on 1814/1841 datapoints.

For each baseline model, we evaluated the area under the curve of the receiver operating characteristic and precision-recall curves (ROC-AUC, PR-AUC). Notably, Elevation and CRISTA are regression models whereas we compared classification performance. Sweeping thresholds in the evaluation of ROC-AUC and PR-AUC can standardize such comparisons. We evaluated our own model architectures using five different initial random seeds and performed a one sample $z$-test relative to the AUCs of the baselines.

\subsection{Model Training and Validation}

We aim to convey the value of the TrueOT dataset and its underlying distinction from data generated by proxy assays. Therefore, we train and validate our own models exclusively on the Proxy Dataset. Throughout this work, splitting data is an approximate process due to the need to keep gRNAs unique in each subset of data. We performed training and five-fold cross validation (CV) on approximately $80 \%$ of the Proxy Dataset ("Proxy TrainCV"). Within each fold, we apply an inverse class weight to account for class imbalance. 


\begin{tabular}{|l|c|c|c|c|c|l|}
\hline Dataset Name & Original $n$ & gRNAs & $n$ & Bulge & Other & Purpose \\
\hline TrueOT & 1841 & 36 & 1841 & 227 & 1614 & Holdout Dataset \\
\hline TrueOT_CNN_std & & 18 & 1059 & 0 & 1059 & \\
TrueOT_Elevation & & 26 & 1118 & 0 & 1118 & \\
TrueOT_predictCRISPR & 1841 & 12 & 857 & 0 & 857 & Pairwise Comparisons \\
TrueOT_CRISTA & & 25 & 1252 & 186 & 1066 & \\
TrueOT_CRISPR-NET & & 15 & 1078 & 182 & 896 & \\
\hline Proxy Dataset & 18072 & 31 & 3505 & 157 & 3348 & Developing SCNNs \\
\hline Proxy TrainCV & n/a & 23 & 2811 & 129 & 2682 & Training/CV \\
Proxy Validation & n/a & 8 & 694 & 28 & 666 & Hyperparameter Selection \\
\hline
\end{tabular}

Table 1: Summary of datasets. TrueOT was used as a holdout evaluation set, and subsets were used in pairwise comparisons against published baseline algorithms with $n$ denoting the number of datapoints in each set. The Proxy Dataset was used for internal model development with cross-validation (CV) ensembling on Proxy TrainCV and hyperparameter selection guided by Proxy Validation.

We conducted majority vote CV ensembling with early stopping based on CV performance for each model architecture. The ensembling process helps each member model learn to generalize to a different subset of gRNAs in Proxy TrainCV. Performance on these five folds is too variable to inform hyperparameter selection due to the limited unique gRNAs in each fold. Therefore, we use the ensemble ROC-AUC on the remaining $20 \%$ of data ("Proxy Validation") as a far more stable guide. This decision also reflects the common practice of using generalization on proxy datasets as a ranking mechanism of scoring algorithms.

\subsection{Siamese Model Design}

We selected a Siamese network as our core architecture which is commonly used for sequence comparisons in natural language processing with some recent adaptations to biological settings [44, 45]. A Siamese network passes elements of paired data through an identical sequence processing network and evaluates their similarity. Here, we assessed the Euclidean distance between the gRNA and target sequences' network output. By optimizing a contrastive loss function, Siamese networks learn to position similar sequences (i.e., gRNA-target pair that results in editing) close together while pushing dissimilar pairs further apart. A Siamese network is particularly helpful for investigating the influence of various network depths because its output dimension is arbitrary; layers can easily be added or removed without having to condense the final output to a scalar value (regression) or the number of classes (classification). Such dimensionality reduction in other neural networks is often accomplished by dense layers which are parameter intensive and unlikely to transfer well between datasets.

\subsubsection{Data Encoding}

Existing bulge-aware scoring algorithms require alignment for data encoding 19, 20]. Because alignment algorithms can vary in output given the same pair of sequences, scoring algorithms may be susceptible to these disagreements. We simplify the encoding strategy to ignore exact alignment and attempt to correct for small frameshifts caused by bulges through a max pooling operation. The gRNA and target nucleotides $\{\mathrm{A}, \mathrm{C}, \mathrm{G}, \mathrm{T} / \mathrm{U}\}$ are one-hot encoded, and by simply left-padding the sequences with zeros to a fixed length of 26, our encoding is alignment-independent (Fig. 1a). We chose 26 as up to three insertions have been allowed in past alignment techniques for CRISPR scoring 20 .

\subsubsection{Siamese Networks}

We start with a single convolutional and pooling layer with many filters because of such layers' established ability to capture features that generalize across datasets 32. This Siamese 1-Convolution model (S1C) anomalously allows filters to be added indefinitely without harming generalization (in theory) since the model's output dimension is arbitrary and a distance is immediately computed after pooling. As a result, we use $2^{14}=16384$ filters of 8 nucleotide width in our S1C based on our hyperparameter search (Fig. S2). For bulge awareness, we add a $1 \times 2$ max pooling operator with stride 1 , allowing our network to partially 


\section{\begin{tabular}{|l|l|l|l|l|l|l|l|l|l}
\hline- & - & - & G & G & C & A & C & T & $\cdots$ \\
\hline
\end{tabular}}
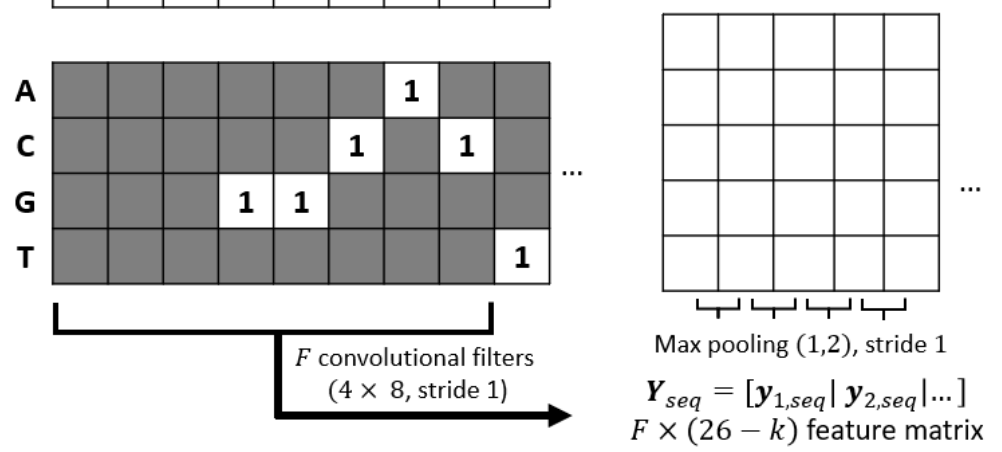

$$
D=\left\|\boldsymbol{Y}_{g R N A}-\boldsymbol{Y}_{D N A}\right\|_{\boldsymbol{F}}
$$

(a) $\mathrm{S} 1 \mathrm{C}$

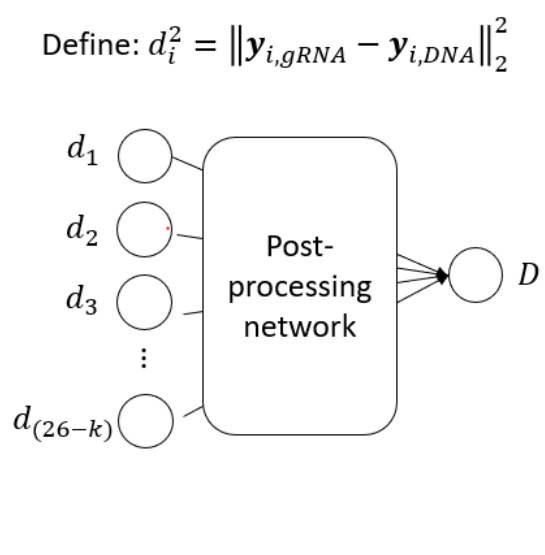

(b) Hybrid S1C

Figure 1: The Siamese models compute a distance $D$ between a guide RNA (gRNA) and target DNA sequence. (a) Rather than aligning the gRNA and target sequences, we simply left-pad them to a fixed length of 26 . The S1C applies a single convolutional and pooling layer to each one-hot encoded sequence. The filter vector at position $i$ for a sequence $s e q$ is denoted $y_{i, s e q}$. The distance is the standard Euclidean distance between the feature matrices of the gRNA and DNA sequences. (b) The Hybrid S1C computes the squared distance between two input sequences at each position from the output of an S1C. Note that the S1C's distance can be represented by $D=\sqrt{\mathbf{1}^{T} d_{i}^{2}}$. By passing the position-wise distances to a post-processing network with a nonnegative activation function (e.g., ReLU), the Hybrid S1C can learn a nonlinear "distance" guided by a contrastive loss function. The post-processing network is entirely arbitrary in principle, although we use a single dense layer of 256 neurons in this work.

tolerate \pm 1 position frameshifts of sequences. We also hypothesize that our relatively wide 8 -nucleotide convolutions will learn some frameshift tolerance themselves.

Despite these features, there are several reasons why additional model complexity is warranted. First, the S1C only measures local dependencies within 8 nucleotide windows. By computing a distance on the output of this layer, neither positions nor combinations of features are considered, both of which have established relevance 6, 13. There are arbitrarily many ways to increase model complexity, but we present just two simple cases representative of the evidence of a need for transfer learning. In one implementation, we add a second convolutional and pooling layer (S2C), and in a second, we add a dense layer (S1C_Dense). Unlike in S1C, we constrain the number of filters (256) in the first convolutional layer for these deeper models to prevent an explosion of parameters. To directly compare S2C and S1C_dense against a single convolutional layer, we define the S1C_mini model with just these 256 filters.

\subsubsection{Untrained S1C}

Random kernel weights in convolutions have been shown to create distance-preserving ("isometric") embeddings of inputs 46 47], making them a natural choice for a Siamese network that makes decisions based on the output positions of input sequences. Training kernel weights distorts the network's output positions to better separate classes, especially with datapoints at the boundary [47]. In the context of CRISPR scoring, we define boundary points as gRNA-target pairs with strong sequence homology that do not result in editing and vice versa. We suspect that comparing the performance of an untrained S1C model ("S1C_ut") against that of the trained Siamese models and existing baselines may provide insight into the influence of training on the resolution of boundary points.

\subsubsection{Hybrid S1C for Transfer Learning}

In applying transfer learning, we use the S1C trained on the Proxy Dataset as a pretrained base network with frozen weights, add on a post-processing network, and train the latter network's weights on roughly half of TrueOT (Fig. 1b). Note that TrueOT is a very small dataset such that the post-processing network must be 
relatively simple for any meaningful training to occur, but the S1C output dimension is very high. However, the $\mathrm{S} 1 \mathrm{C}$ is position-invariant with an output equivalent to the square root of the sum of the squared position differences in each convolutional window (Fig. 1b). This intuition lends a natural way to dramatically reduce the output dimension of the S1C for transfer learning on a small dataset: we compute the squared position differences and pass this small vector to a dense network. With a ReLU activation on the final output, this "Hybrid S1C" learns a nonlinear "distance" metric that accounts for combinations of positions of sequence discrepancies between the gRNA and target. We remove the square root function on the output since it is a monotonic function and ROC and PR characterizations are based on thresholds. We initialize the weights such that the model starts as an equivalent classifier to the S1C.

We split TrueOT approximately into $75 \%$ (TrueOT Train CV) for training and CV and $25 \%$ (TrueOT Test) for testing, enforcing non-overlapping gRNAs between each split. We performed a similar five-fold CV training and ensembling process described in Section 2.3. For each fold of the Hybrid S1C, we loaded the weights of the corresponding fold from the original S1C and froze them, trained only the additional dense layers of the post processing network on a portion of the TrueOT training set, and used the CV portion to guide early stopping. We used CV performance for hyperparameter selection, ultimately choosing a single hidden layer of 256 neurons. This process maintained the Hybrid S1C as a five-member ensemble.

To evaluate the effect on TrueOT Test performance, we made two versions of the Hybrid S1C. For the first, we used the transfer learning process described above. For the second, we trained the entire network, including the convolutional layer, on Proxy TrainCV. We used random weight initializations and selected that with the best Proxy Validation performance. We generated ten different splits of TrueOT and evaluated the change in ROC-AUC performance relative to the original S1C. Repeated splitting of TrueOT ensures that any apparent performance change is not just an anomaly of a particular split. Comparing these two versions of the Hybrid S1C indicates whether the performance change is due to the transfer learning process specifically (freezing weights from the original S1C) or merely due to the change in network architecture.

\section{Results}

\subsection{Model Design by Proxy Validation Compromises TrueOT Performance}

Figure2 2 illustrates a general pattern from our research: improving generalization performance on the Proxy Validation set can compromise the performance on TrueOT. The untrained network performs slightly better than guessing on the Proxy Validation set (ROC-AUC 0.626, PR-AUC 0.250) but substantially better on TrueOT (ROC-AUC 0.797, PR-AUC 0.482), meaning that classes of sequence pairs in TrueOT are more closely related to an absolute count of mismatches along with some frameshift tolerance. Although we have no influence over the distribution of data labels in TrueOT, this contrast illustrates that our Proxy Validation set has many more boundary points from which our Siamese models may hope to learn from.

In adding subsequent layers to our Siamese models, recall that S1C_dense and S2C are built off of S1C_mini. These simple extensions dramatically improve performance on the Proxy Validation set while exhibiting worse performance on TrueOT than S1C_mini (Fig. 22). This pattern holds for both ROC-AUC and PR-AUC. Ultimately, these results indicate that the Proxy Validation set is suboptimal for guiding model design if the goal is to generalize to TrueOT. We speculate that this is caused by an underlying discrepancy in the biology of proxy assays versus in vivo editing. If the two datasets were from the same underlying distributions, this inverse effect would be very unlikely to occur. Moreover, the two best-performing models on TrueOT are S1C and S1C_mini. Interestingly, the many extra filters in S1C substantially improve the Proxy Validation performance over S1C_mini, but both of these single-convolutional models maintain similar TrueOT performance. This result is consistent with the established robustness of high level convolutional layers in transfer learning applications.

\subsection{S1C Achieves State-of-the-Art Performance on TrueOT}

Here we compare the S1C against baselines given the results in Section 3.1 and include S1C_ut as a useful reference. In pairwise comparisons on appropriate subsets of TrueOT, our S1C significantly outperforms most of the tested baseline algorithms (Table 2). Two exceptions are Elevation and predictCRISPR, which outperform the S1C. However, these models cannot account for bulges, meaning that this comparison is 


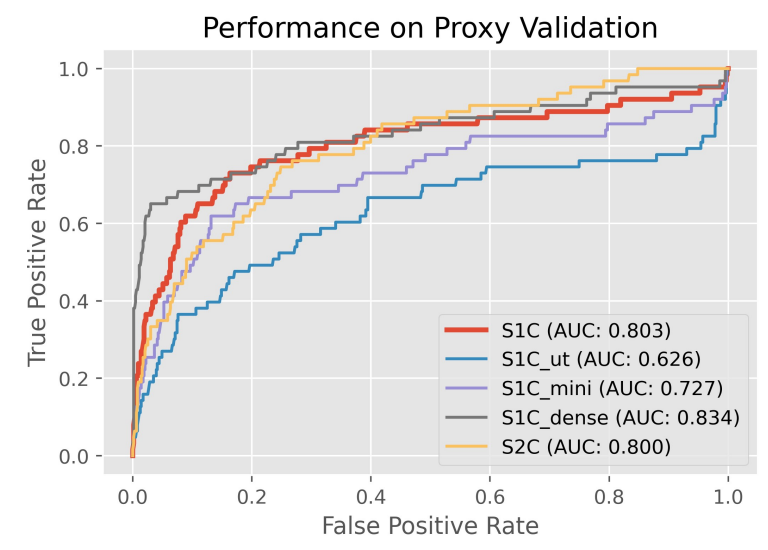

(a)

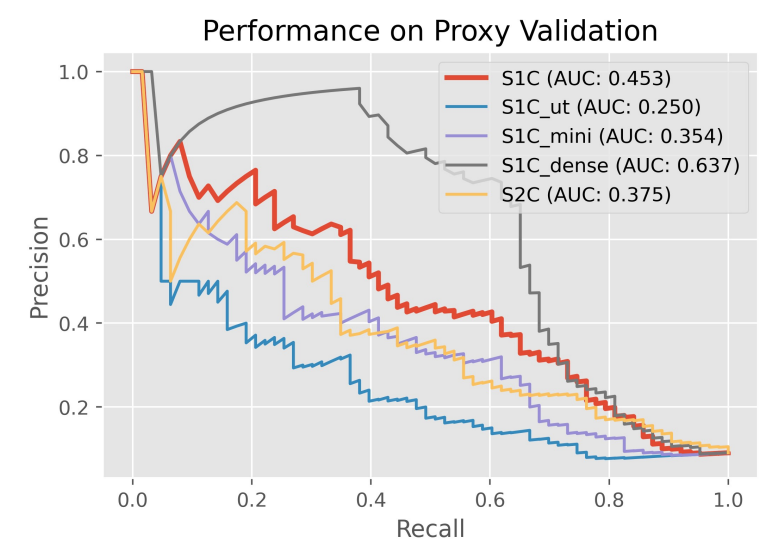

(c)

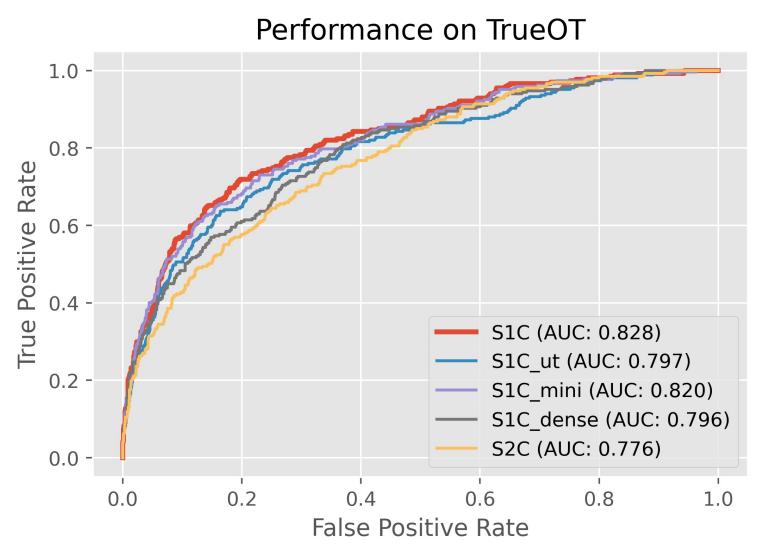

(b)

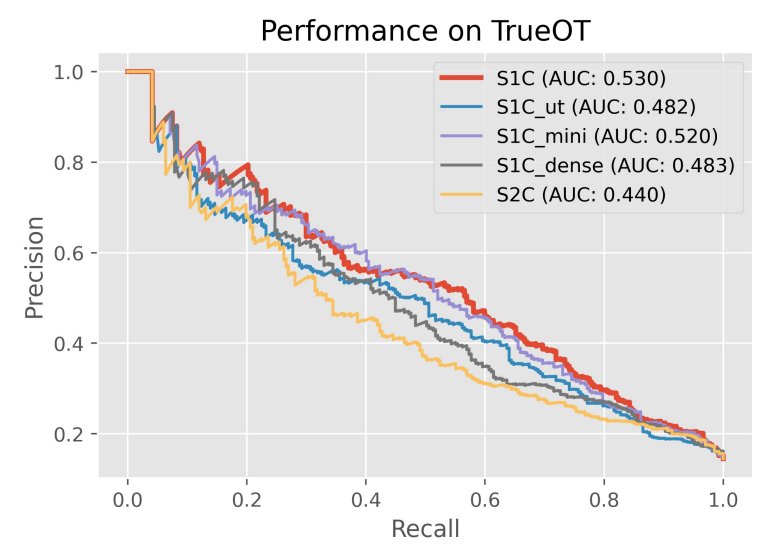

(d)

Figure 2: Comparison of generalization performance on Proxy Validation and TrueOT for various Siamese models. (a,b) Comparison of ROC-AUC (c,d) Comparison of PR-AUC.

restricted to gRNA-target pairs of equal length. The S1C appears roughly on par with CRISPR-NET, slightly underperforming in ROC-AUC and outperforming in PR-AUC.

Among all baselines, COSMID, CRISTA, and CRISPR-NET are designed to account for bulges. We further partitioned the TrueOT into bulge-containing and bulge-excluded datasets to deepen our comparison against these models (Table 3). The S1C significantly outperforms the baselines in almost all categories. One exception is a larger $p$ value $(p=0.022)$ in the PR-AUC comparison against COSMID for bulge-containing datapoints. The second exception appears to clarify the comparable overall performance of the S1C and CRISPR-NET in Table 2 CRISPR-NET has an edge on bulge-excluded datapoints while the S1C has better performance on bulge-containing datapoints.

The S1C's approximate equivalence with the state-of-the-art should not be taken lightly. By including only a single convolutional layer, the S1C has no capacity to learn nonlinear combinations of features, unlike all of the ML-based methods noted here. Moreover, even the untrained S1C_ut outperforms most rulebased and some ML models. The S1C_ut essentially counts the number of mismatches with some frameshift tolerance. While more patterns are clearly necessary to distinguish boundary points in a dataset, it appears that the added functional capacity of existing models does not necessarily improve in vivo editing predictions. 
bioRxiv preprint doi: https://doi.org/10.1101/2021.08.28.457846; this version posted August 29, 2021. The copyright holder for this preprint (which was not certified by peer review) is the author/funder, who has granted bioRxiv a license to display the preprint in perpetuity. It is made available under aCC-BY-NC 4.0 International license.

\begin{tabular}{|l|c|c|c|c|c|c|c|}
\hline \multicolumn{2}{|c|}{} & \multicolumn{3}{|c|}{ ROC-AUC } & \multicolumn{3}{c|}{ PR-AUC } \\
\hline Baseline Name & $n$ & Baseline & S1C & S1C_ut & Baseline & S1C & S1C_ut \\
\hline Cropit & 1614 & 0.686 & $\mathbf{0 . 8 2 1}$ & $\mathbf{0 . 7 8 6}$ & 0.352 & $\mathbf{0 . 5 4 2}$ & $\mathbf{0 . 4 9 2}$ \\
\hline Hsu & 1614 & 0.668 & $\mathbf{0 . 8 2 1}$ & $\mathbf{0 . 7 8 6}$ & 0.369 & $\mathbf{0 . 5 4 2}$ & $\mathbf{0 . 4 9 2}$ \\
\hline CCTop & 1614 & 0.662 & $\mathbf{0 . 8 2 1}$ & $\mathbf{0 . 7 8 6}$ & 0.341 & $\mathbf{0 . 5 4 2}$ & $\mathbf{0 . 4 9 2}$ \\
\hline MIT & 1614 & 0.766 & $\mathbf{0 . 8 2 1}$ & $\mathbf{0 . 7 8 6}$ & 0.440 & $\mathbf{0 . 5 4 2}$ & $\mathbf{0 . 4 9 2}$ \\
\hline CFD & 1614 & 0.813 & $\mathbf{0 . 8 2 1}$ & 0.786 & 0.492 & $\mathbf{0 . 5 4 2}$ & 0.492 \\
\hline COSMID & 1819 & 0.669 & $\mathbf{0 . 8 2 6}$ & $\mathbf{0 . 7 9 5}$ & 0.350 & $\mathbf{0 . 5 3 3}$ & $\mathbf{0 . 4 8 3}$ \\
\hline CNN_std & 1059 & 0.724 & $\mathbf{0 . 8 1 6}$ & $\mathbf{0 . 7 6 2}$ & 0.426 & $\mathbf{0 . 5 0 3}$ & 0.422 \\
\hline Elevation & 1118 & $\mathbf{0 . 7 9 9}$ & 0.784 & 0.731 & $\mathbf{0 . 4 4 2}$ & 0.413 & 0.374 \\
\hline predictCRISPR & 857 & $\mathbf{0 . 8 1 0}$ & 0.756 & 0.701 & $\mathbf{0 . 3 9 6}$ & 0.359 & 0.318 \\
\hline CRISTA & 1252 & 0.731 & $\mathbf{0 . 7 7 6}$ & 0.731 & 0.354 & $\mathbf{0 . 3 9 0}$ & 0.352 \\
\hline CRISPR-NET & 1078 & $\mathbf{0 . 7 7 9}$ & 0.755 & 0.704 & 0.198 & $\mathbf{0 . 3 2 9}$ & $\mathbf{0 . 2 8 6}$ \\
\hline
\end{tabular}

Table 2: Pairwise Comparisons on TrueOT. We conduct one-sided $z$-tests between the Baselines and the S1C's. Baselines' AUCs are bolded if they are greater than both the S1C's and S1C_ut's AUCs with $p<0.001$. The S1Cs' AUCs are bolded if they are greater than the Baselines' AUC with $p<0.001$.

\begin{tabular}{|l|l|c|c|c|c|c|c|c|}
\hline \multicolumn{2}{|c|}{} & \multicolumn{3}{|c|}{ ROC-AUC } & \multicolumn{3}{c|}{ PR-AUC } \\
\hline Baseline Name & TrueOT Subset & $n$ & Baseline & S1C & S1C_ut & Baseline & S1C & S1C_ut \\
\hline \multirow{2}{*}{ COSMID } & Other & 1614 & 0.656 & $\mathbf{0 . 8 2 1}$ & $\mathbf{0 . 7 8 6}$ & 0.358 & $\mathbf{0 . 5 4 2}$ & $\mathbf{0 . 4 9 2}$ \\
\cline { 2 - 10 } & Bulge & 205 & 0.733 & $\mathbf{0 . 8 2 0}$ & $\mathbf{0 . 8 0 8}$ & 0.279 & 0.336 & $\mathbf{0 . 3 3 8}$ \\
\hline \multirow{2}{*}{ CRISTA } & Other & 1066 & 0.739 & $\mathbf{0 . 7 7 5}$ & 0.721 & 0.374 & $\mathbf{0 . 4 0 5}$ & 0.366 \\
\cline { 2 - 9 } & Bulge & 186 & 0.584 & $\mathbf{0 . 8 1 0}$ & $\mathbf{0 . 8 0 3}$ & 0.089 & $\mathbf{0 . 3 3 5}$ & $\mathbf{0 . 3 4 0}$ \\
\hline \multirow{2}{*}{ CRISPR-NET } & Other & 896 & $\mathbf{0 . 8 4 4}$ & 0.749 & 0.721 & $\mathbf{0 . 3 5 9}$ & 0.342 & 0.297 \\
\cline { 2 - 9 } & Bulge & 182 & 0.643 & $\mathbf{0 . 8 0 6}$ & $\mathbf{0 . 8 0 3}$ & 0.073 & $\mathbf{0 . 3 3 5}$ & $\mathbf{0 . 3 4 0}$ \\
\hline
\end{tabular}

Table 3: Pairwise Comparisons on TrueOT for bulge-capable models. The original $n$ datapoints available for pairwise comparisons were split into bulge-containing gRNA-target pairs and all other pairs. A pair was considered to have a bulge if a '-' appeared in either sequence in the original study's alignment or if the two sequences were of different lengths. Bold font is applied as in Table 2 .

\subsection{Baseline Models' Datasets Reflect their TrueOT Performance}

Our pairwise model evaluations are intended to compare baselines against the S1C and do not directly reflect a rank ordering among baselines. However, the relative performance appears consistent with our transfer learning hypothesis; better performing baselines incorporate more in vivo-based data in their training process. PredictCRISPR used many of the low-throughput datapoints contained in TrueOT in its training set [18, hence its fewest datapoints $n$ on which we can perform a pairwise comparison fairly. CFD performs the best among the rule-based algorithms on the full 1614 bulge-excluded datapoints and comes very close to the performance of the S1C. We suggest that while CFD is often labeled as rule-based in the literature, it could be considered an ML approach driven by in vivo data as its weights are tuned based on flow cytometry data [6]. These direct in vivo measurements, while not based on sequence modification rate for inclusion in TrueOT, are arguably very close in motivation. Lastly, Elevation derived its model as a generalization of CFD, which may explain its similarly high performance. More details on the datasets used in each ML baseline are available in Table S3.

\subsection{Bulge Performance of the S1C Appears Driven by Max Pooling}

The S1C's superior performance on the bulge-containing subsets of TrueOT (Table 3) could be due to chance given the small number of datapoints available $(n \leq 205)$. Nonetheless, we investigated the decision-making process of the $\mathrm{S} 1 \mathrm{C}$ for bulges to understand its high performance. We hypothesized that the use of many filters $\left(2^{14}\right)$ allowed the S1C to treat mismatches due to small frameshifts from bulges differently than random mismatches. For example, an insertion at position 4 in a window on the target sequence could appear as 5 mismatches with gRNA (Fig. 3a), but perhaps the S1C learns to recognize such an occurrence as a frameshift 


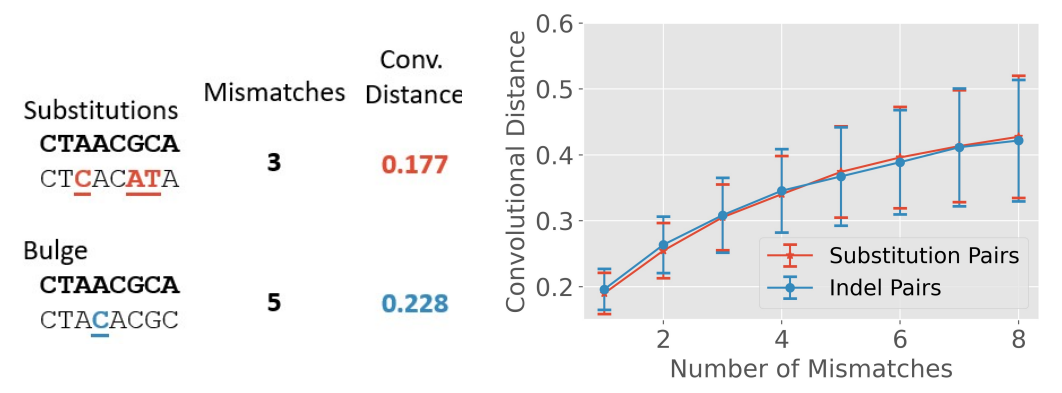

(a) Example Datapoint

(b) $\mathrm{S} 1 \mathrm{C}$

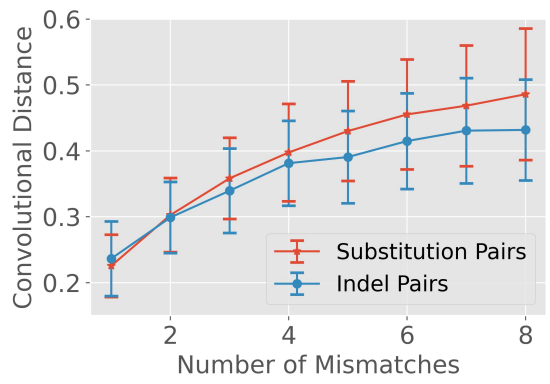

(c) S1C Without Pooling

Figure 3: Characterization of the capacity of convolutions to learn the effect of bulges. Error bars \pm 1 s.d. (a) Pairs of sequences were generated either by introducing substitutions or bulges at specific positions. For each pair in each method of mutation, the raw number of mismatches and convolutional distance was evaluated. (b) Comparison of the relationship between mismatches and network distance for the S1C. (c) Same comparison with an S1C without pooling.

instead.

We tested this hypothesis by generating a series of random sequences and pairing them either with a mutated sequence with a random number of mismatches or with a sequence modified by an insertion or deletion. To maintain a length of 8 for an insertion event, the 3' base was truncated; for a deletion, a random base was appended to the 3' end. We evaluated the distances computed by the S1C's filters between each pair of sequences and plotted them as a function of the naive mismatch count. While we expected the bulge-generated pairs to exhibit lower convolutional distances than the mismatch-generated pairs - an indication of recognizing greater similarity - we were surprised to find essentially the same distribution of distances for both sets of sequences (Fig. 3b. The distance computation between pairs appears unaware of bulges, indicating that the $\mathrm{S} 1 \mathrm{C}$ is managing bulges predominantly through max pooling. Indeed, the untrained S1C_ut performs similarly on bulge datapoints as the S1C (Table 3) and is only utilizing max pooling for bulge awareness. When we remove pooling and retrain the S1C with an otherwise identical network, the filters are forced to learn to recognize bulges on their own (Fig. 3c).

Max pooling is indifferent to the type and sequence neighborhood of a bulge by merely allowing for \pm 1 shift in the position of sequence features. This limited handling of bulges in the S1C outperforms existing algorithms on bulge datapoints and supports the notion of underlying discrepancies between proxy datasets and TrueOT. Learning the influence of bulge type and neighborhood is the role of model training. We speculate that much like our results in Section 3.1. existing models may have appeared to improve performance on the validation and test sets used by the respective authors while resulting in reduced performance on TrueOT.

\subsection{Transfer Learning with Hybrid S1C Improves TrueOT Generalization}

In evaluating the effect on ROC-AUC for ten different splits of TrueOT (Fig. 4), we find that training the dense layers of the Hybrid S1C on a portion of TrueOT TrainCV while freezing the convolution weights from the original S1C slightly improves the ensemble performance on TrueOT Test (one-sided paired t-test, $p=0.047)$. We speculate that the small improvement is mostly due to limited training data in splits of TrueOT TrainCV. Training the full Hybrid S1C network from scratch on the Proxy TrainCV achieved the best Proxy Validation performance in this study (ROC-AUC 0.877), a substantial improvement over the original S1C (ROC-AUC 0.803, Fig. 2a). However, Figure 4 shows that its performance on TrueOT Test splits is significantly lower than that of the original S1C $(p=0.0004)$. This contrasting result echoes that of Figure 2 and further confirms that Proxy Validation is not appropriate to guide model design. Indeed, when training the Hybrid S1C from scratch, some initializations that resulted in poorer Proxy Validation generalization had marginally improved TrueOT Test performance (results not shown). Ultimately, the Hybrid S1C architecture reliably improves TrueOT Test performance when applied through a transfer learning framework. Future 


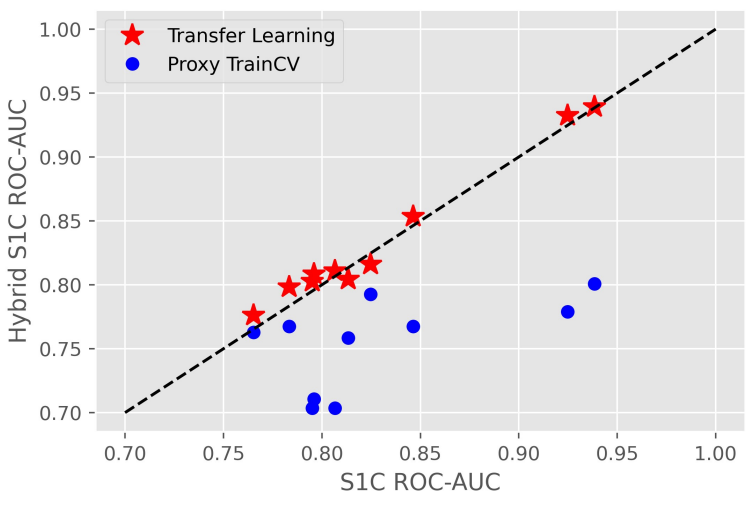

Figure 4: Performance comparison of two versions of the Hybrid S1C against the original S1C on ten different splits of TrueOT Test. For the "Transfer Learning" datapoints (red stars), the original S1C model's weights, previously trained on the Proxy TrainCV, were loaded into the Hybrid S1C and frozen. The Hybrid S1C's dense parameters were then trained on TrueOT TrainCV. This framework lends a slight overall improvement over the original S1C ( $p=0.047$ in one-sided paired t-test). For comparison (blue circles), the full Hybrid S1C architecture was trained from scratch on Proxy TrainCV with various initial random seeds. Selecting the initialization that lends the highest performance on Proxy Validation results in reduced generalization performance on the TrueOT Test sets $(p=0.0004)$.

research may see larger performance gains as more data is available for inclusion in TrueOT for tuning deeper layers.

\section{Discussion}

The computational scoring of putative OTs is essential for economically designing safe gRNAs. Scoring models to date have reported test set performance on datasets sourced by assays that measure a proxy of $i n$ vivo editing. Each of these sources carries the risk of being misaligned with true in vivo editing behavior. Models should be evaluated based on their ability to predict the in vivo editing behavior of gRNA sequences that were unseen during model training [48. We suggest that our curated TrueOT dataset offers a starting point for an accepted benchmark, and our initial exploration towards a new model supports our hypothesis of an underlying mismatch between various datasets.

Our simple S1C model with only one convolutional layer achieves state-of-the-art performance on TrueOT as reflected by pairwise comparisons against several rule-based and ML models. This result suggests the potency of a Siamese convolutional architecture for deriving generalizable features across datasets. Moreover, adding subsequent layers to S1C can improve generalization performance on our Proxy Validation set while compromising performance on TrueOT. This inverse relationship strongly suggests that the data in the two datasets originate from different distributions; sequence features that govern editing in vivo are sufficiently distinct from those of the assays represented in Proxy Validation.

We are concerned that an underlying discrepancy among datasets is a pervasive issue in the CRISPR scoring literature. Notably, even the untrained S1C performed comparatively if not better than many baseline models on TrueOT. This result indicates that baselines may have learned irrelevant (if not misleading) features for TrueOT during training on data from proxy assays, an effect we observed during our own development of Siamese networks (Figs. 2, 44. While researchers commonly use one or a few external datasets for model comparisons, there is little consideration of which dataset is more reflective of in vivo editing. Therefore, we believe there is an urgent need for a common benchmark dataset such as TrueOT to compare models. We acknowledge that this work does not consider the influences of epigenetic features that can lend variable behavior between cell types. As more in vivo-validated data becomes available, we envision TrueOT being split into nuanced subsets of data for particular applications.

If training data is sourced from proxy assays, transfer learning principles should be considered for improving performance on such benchmark datasets. Our S1C's high performance and extreme simplicity offers a strong baseline against which to compare new models and a potent starting point for extended research as there is substantial room for improvement. A single convolutional layer aggregates local relationships 
without considering position or nonlinear combinations of features. Added complexity directly to the S1C as posed here will be difficult with its abnormally high $\left(2^{14}\right)$ number of filters (an idiosyncrasy of the S1C architecture). However, either the S1C_mini with far fewer $\left(2^{8}\right)$ filters or the dimensionality reduction offered by the Hybrid S1C framework could serve as more accessible starting points. In any case, our results suggest that benefiting from additional model complexity will be increasingly feasible as data is added to TrueOT and transfer learning approaches are considered.

We are optimistic that the expansion of TrueOT and applied transfer learning principles will help alleviate other issues in CRISPR datasets by better elucidating the causes of OT activity. For instance, it is widely assumed that the targets with low sequence homology to a gRNA will have zero editing efficiency such that in many assays, signals from such target sites are treated as noise and excluded from the final output [26 49]. These exclusions may include datapoints with bulges depending on the particular alignment method used to gauge sequence homology [20. While the field gradually recognizes the importance of OTs with bulges, the noise filtering process based on homology carries the risk of mislabeling target sites with bulges. Further experimental validations on sites with low sequence homology will help distinguish genuine OTs from noise generated by somatic mutations and DNA repairs while also benefiting the basic research of gRNA-target hybridization mechanism.

In conclusion, there is an unmet need for a widely accepted gold standard dataset for benchmarking OT evaluation pipelines. While we propose the TrueOT dataset and its corresponding inclusion criteria, we urge the CRISPR community to more broadly recognize the need for such a dataset and modify TrueOT as it sees fit. We find substantial evidence that the datasets used in model development should not be considered equivalent from a machine learning perspective. Instead, they appear to have discrepancies such that the decision-making processes learned from one dataset may transfer poorly to another. As more experimentally validated in vivo editing data becomes available, dedicated transfer learning efforts can begin to properly leverage the quantity of high-throughput data.

\section{Acknowledgments}

This work was supported by the National Institutes of Health (UG3HL151545 and R01HL152314 to G.B.) and a Vannevar Bush Faculty Fellowship (ONR grant N00014-18-1-2047 to R.G.B.). P.K.K. was supported by the NLM Training Program in Biomedical Informatics and Data Science (T15LM007093).

\section{References}

[1] K. M. Esvelt, P. Mali, J. L. Braff, M. Moosburner, S. J. Yaung, and G. M. Church, "Orthogonal Cas9 proteins for RNA-guided gene regulation and editing," Nat. Methods, vol. 10, no. 11, pp. 1116-1121, 2013.

[2] P. Mali, L. Yang, K. M. Esvelt, J. Aach, M. Guell, J. E. DiCarlo, J. E. Norville, and G. M. Church, "RNA-guided human genome engineering via Cas9," Science, vol. 339, no. 6121, pp. 823-826, 2013.

[3] P. D. Hsu, E. S. Lander, and F. Zhang, "Development and applications of CRISPR-Cas9 for genome engineering," Cell, vol. 157, no. 6, pp. 1262-1278, 2014.

[4] F. A. Ran, P. D. Hsu, J. Wright, V. Agarwala, D. A. Scott, and F. Zhang, "Genome engineering using the CRISPR-Cas9 system," Nat. Protoc., vol. 8, no. 11, pp. 2281-2308, 2013.

[5] Y. Fu, J. A. Foden, C. Khayter, M. L. Maeder, D. Reyon, J. K. Joung, and J. D. Sander, "Highfrequency off-target mutagenesis induced by CRISPR-Cas nucleases in human cells," Nat. Biotechnol., no. 9, pp. 822-826, 2013.

[6] J. G. Doench, N. Fusi, M. Sullender, M. Hegde, E. W. Vaimberg, K. F. Donovan, I. Smith, Z. Tothova, C. Wilen, R. Orchard et al., "Optimized sgRNA design to maximize activity and minimize off-target effects of CRISPR-Cas9," Nat. Biotechnol., vol. 34, no. 2, pp. 184-191, 2016. 
[7] J. Zischewski, R. Fischer, and L. Bortesi, "Detection of on-target and off-target mutations generated by CRISPR/Cas9 and other sequence-specific nucleases," Biotechnol. Adv., vol. 35, no. 1, pp. 95-104, 2017.

[8] T. J. Cradick, E. J. Fine, C. J. Antico, and G. Bao, "CRISPR/Cas9 systems targeting $\beta$-globin and ccr5 genes have substantial off-target activity," Nucleic Acids Res., vol. 41, no. 20, pp. 9584-9592, 2013.

[9] S. W. Cho, S. Kim, Y. Kim, J. Kweon, H. S. Kim, S. Bae, and J.-S. Kim, "Analysis of off-target effects of CRISPR/Cas-derived RNA-guided endonucleases and nickases," Genome Res., vol. 24, no. 1, pp. $132-141,2014$.

[10] M. Haeussler, K. Schönig, H. Eckert, A. Eschstruth, J. Mianné, J.-B. Renaud, S. Schneider-Maunoury, A. Shkumatava, L. Teboul, J. Kent et al., "Evaluation of off-target and on-target scoring algorithms and integration into the guide RNA selection tool CRISPOR," Genome Biol., vol. 17, no. 1, pp. 1-12, 2016.

[11] J. Listgarten, M. Weinstein, B. P. Kleinstiver, A. A. Sousa, J. K. Joung, J. Crawford, K. Gao, L. Hoang, M. Elibol, J. G. Doench, and N. Fusi, "Prediction of off-target activities for the end-to-end design of CRISPR guide RNAs," Nat. Biomed. Eng., vol. 2, no. 1, pp. 38-47, 2018.

[12] F. Heigwer, G. Kerr, and M. Boutros, "E-CRISP: fast CRISPR target site identification," Nat. Methods, vol. 11, no. 2, pp. 122-123, 2014.

[13] T. J. Cradick, P. Qiu, C. M. Lee, E. J. Fine, and G. Bao, "COSMID: a web-based tool for identifying and validating CRISPR/Cas off-target sites," Mol. Ther. Nucleic Acids, vol. 3, p. e214, 2014.

[14] M. Stemmer, T. Thumberger, M. del Sol Keyer, J. Wittbrodt, and J. L. Mateo, "CCTop: an intuitive, flexible and reliable CRISPR/Cas9 target prediction tool," PloS one, vol. 10, no. 4, p. e0124633, 2015.

[15] P. D. Hsu, D. A. Scott, J. A. Weinstein, F. A. Ran, S. Konermann, V. Agarwala, Y. Li, E. J. Fine, X. Wu, O. Shalem et al., "DNA targeting specificity of RNA-guided Cas9 nucleases," Nat. Biotechnol., vol. 31, no. 9, pp. 827-832, 2013.

[16] M. W. Libbrecht and W. S. Noble, "Machine learning applications in genetics and genomics," Nat. Rev. Genet., vol. 16, no. 6, pp. 321-332, 2015.

[17] J. Lin and K.-C. Wong, "Off-target predictions in CRISPR-Cas9 gene editing using deep learning," Bioinformatics, vol. 34, no. 17, pp. i656-i663, 2018.

[18] H. Peng, Y. Zheng, Z. Zhao, T. Liu, and J. Li, "Recognition of CRISPR/Cas9 off-target sites through ensemble learning of uneven mismatch distributions," Bioinformatics, vol. 34, no. 17, pp. i757-i765, 2018.

[19] J. Lin, Z. Zhang, S. Zhang, J. Chen, and K.-C. Wong, "CRISPR-Net: A recurrent convolutional network quantifies CRISPR off-target activities with mismatches and indels," Adv. Sci., vol. 7, no. 13, p. 1903562, 2020 .

[20] S. Abadi, W. X. Yan, D. Amar, and I. Mayrose, "A machine learning approach for predicting CRISPRCas9 cleavage efficiencies and patterns underlying its mechanism of action," PLoS Comput. Biol., vol. 13, no. 10, pp. 1-24, 2017.

[21] Y. Lin, T. J. Cradick, M. T. Brown, H. Deshmukh, P. Ranjan, N. Sarode, B. M. Wile, P. M. Vertino, F. J. Stewart, and G. Bao, "CRISPR/Cas9 systems have off-target activity with insertions or deletions between target DNA and guide RNA sequences," Nucleic Acids Res., vol. 42, no. 11, pp. 7473-7485, 2014.

[22] C. R. Lazzarotto, N. L. Malinin, Y. Li, R. Zhang, Y. Yang, G. Lee, E. Cowley, Y. He, X. Lan, K. Jividen et al., "Change-seq reveals genetic and epigenetic effects on CRISPR-Cas9 genome-wide activity," Nat. Biotechnol., vol. 38, no. 11, pp. 1317-1327, 2020. 
[23] F. Martin, S. Sánchez-Hernández, A. Gutiérrez-Guerrero, J. Pinedo-Gomez, and K. Benabdellah, "Biased and unbiased methods for the detection of off-target cleavage by CRISPR/Cas9: an overview," Int. J. Mol. Sci., vol. 17, no. 9, p. 1507, 2016.

[24] K. A. Schaefer, W.-H. Wu, D. F. Colgan, S. H. Tsang, A. G. Bassuk, and V. B. Mahajan, "Unexpected mutations after CRISPR-Cas9 editing in vivo," Nat. Methods, vol. 14, no. 6, p. 547, 2017.

[25] Y. Dong, H. Li, L. Zhao, P. Koopman, F. Zhang, and J. X. Huang, "Genome-wide off-target analysis in CRISPR-Cas9 modified mice and their offspring," G3 (Bethesda), vol. 9, no. 11, pp. 3645-3651, 2019.

[26] S. Q. Tsai, Z. Zheng, N. T. Nguyen, M. Liebers, V. V. Topkar, V. Thapar, N. Wyvekens, C. Khayter, A. J. Iafrate, L. P. Le, M. J. Aryee, and J. K. Joung, "GUIDE-seq enables genome-wide profiling of off-target cleavage by CRISPR-Cas nucleases," Nat. Biotechnol., vol. 33, no. 2, pp. 187-198, 2015.

[27] S. Q. Tsai, N. T. Nguyen, J. Malagon-Lopez, V. V. Topkar, M. J. Aryee, and J. K. Joung, "CIRCLE-seq: a highly sensitive in vitro screen for genome-wide CRISPR-Cas9 nuclease off-targets," Nat. Methods, vol. 14, no. 6, p. 607, 2017.

[28] B. Wienert, S. K. Wyman, C. D. Richardson, C. D. Yeh, P. Akcakaya, M. J. Porritt, M. Morlock, J. T. Vu, K. R. Kazane, H. L. Watry et al., "Unbiased detection of CRISPR off-targets in vivo using DISCOVER-Seq," Science, vol. 364, no. 6437, pp. 286-289, 2019.

[29] R. L. Frock, J. Hu, R. M. Meyers, Y.-J. Ho, E. Kii, and F. W. Alt, "Genome-wide detection of DNA double-stranded breaks induced by engineered nucleases," Nat. Biotechnol., vol. 33, no. 2, pp. 179-186, 2015.

[30] J. D. Gillmore, E. Gane, J. Taubel, J. Kao, M. Fontana, M. L. Maitland, J. Seitzer, D. O'Connell, K. R. Walsh, K. Wood et al., "CRISPR-Cas9 in vivo gene editing for transthyretin amyloidosis," N. Engl. J. Med., 2021.

[31] M. Dehghani, Y. Tay, A. A. Gritsenko, Z. Zhao, N. Houlsby, F. Diaz, D. Metzler, and O. Vinyals, "The benchmark lottery," Jul. 2021.

[32] F. Zhuang, Z. Qi, K. Duan, D. Xi, Y. Zhu, H. Zhu, H. Xiong, and Q. He, "A comprehensive survey on transfer learning," Proc. IEEE, vol. 109, no. 1, pp. 43-76, 2021.

[33] D. Kim, S. Bae, J. Park, E. Kim, S. Kim, H. R. Yu, J. Hwang, J.-I. Kim, and J.-S. Kim, "Digenome-seq: genome-wide profiling of CRISPR-Cas9 off-target effects in human cells," Nat. Methods, vol. 12, no. 3, pp. 237-243, 2015.

[34] X. Wang, Y. Wang, X. Wu, J. Wang, Y. Wang, Z. Qiu, T. Chang, H. Huang, R.-J. Lin, and J.-K. Yee, "Unbiased detection of off-target cleavage by CRISPR-Cas9 and talens using integrase-defective lentiviral vectors," Nat. Biotechnol., vol. 33, no. 2, pp. 175-178, 2015.

[35] D. Kim, S. Kim, S. Kim, J. Park, and J.-S. Kim, "Genome-wide target specificities of CRISPR-Cas9 nucleases revealed by multiplex Digenome-seq," Genome Res., vol. 26, no. 3, pp. 406-415, 2016.

[36] N. Gomez-Ospina, S. G. Scharenberg, N. Mostrel, R. O. Bak, S. Mantri, R. M. Quadros, C. B. Gurumurthy, C. Lee, G. Bao, C. J. Suarez et al., "Human genome-edited hematopoietic stem cells phenotypically correct mucopolysaccharidosis type I," Nat. Commun., vol. 10, no. 1, pp. 1-14, 2019.

[37] S. H. Park, C. M. Lee, D. P. Dever, T. H. Davis, J. Camarena, W. Srifa, Y. Zhang, A. Paikari, A. K. Chang, M. H. Porteus et al., "Highly efficient editing of the $\beta$-globin gene in patient-derived hematopoietic stem and progenitor cells to treat sickle cell disease," Nucleic Acids Res., vol. 47, no. 15, pp. 7955-7972, 2019.

[38] M. Pavel-Dinu, V. Wiebking, B. T. Dejene, W. Srifa, S. Mantri, C. E. Nicolas, C. Lee, G. Bao, E. J. Kildebeck, N. Punjya et al., "Gene correction for SCID-X1 in long-term hematopoietic stem cells," Nat. Commun., vol. 10, no. 1, pp. 1-15, 2019. 
[39] S. Vaidyanathan, A. A. Salahudeen, Z. M. Sellers, D. T. Bravo, S. S. Choi, A. Batish, W. Le, R. Baik, M. P. Kaushik, N. Galper et al., "High-efficiency, selection-free gene repair in airway stem cells from cystic fibrosis patients rescues CFTR function in differentiated epithelia," Cell Stem Cell, vol. 26, no. 2, pp. 161-171, 2020.

[40] J. Shapiro, O. Iancu, A. M. Jacobi, M. S. McNeill, R. Turk, G. R. Rettig, I. Amit, A. Tovin-Recht, Z. Yakhini, M. A. Behlke et al., "Increasing CRISPR efficiency and measuring its specificity in hspcs using a clinically relevant system," Mol. Ther. Methods Clin. Dev., vol. 17, pp. 1097-1107, 2020.

[41] H. Xu, T. Xiao, C.-H. Chen, W. Li, C. A. Meyer, Q. Wu, D. Wu, L. Cong, F. Zhang, J. S. Liu et al., "Sequence determinants of improved CRISPR sgRNA design," Genome Res., vol. 25, no. 8, pp. 1147-1157, 2015.

[42] J. Wang, X. Zhang, L. Cheng, and Y. Luo, "An overview and metanalysis of machine and deep learningbased CRISPR gRNA design tools," RNA Biol., vol. 17, no. 1, pp. 13-22, 2020.

[43] R. Singh, C. Kuscu, A. Quinlan, Y. Qi, and M. Adli, "Cas9-chromatin binding information enables more accurate CRISPR off-target prediction," Nucleic Acids Res., vol. 43, no. 18, p. e118, 2015.

[44] H. Cheng, B. Rao, L. Liu, L. Cui, G. Xiao, R. Su, and L. Wei, "PepFormer: End-to-end transformerbased siamese network to predict and enhance peptide detectability based on sequence only," Anal. Chem., vol. 93, no. 16, pp. 6481-6490, 2021.

[45] W. Zheng, L. Yang, R. J. Genco, J. Wactawski-Wende, M. Buck, and Y. Sun, "SENSE: Siamese neural network for sequence embedding and alignment-free comparison," Bioinformatics, vol. 35, no. 11, pp. 1820-1828, 2019.

[46] A. C. Gilbert, Y. Zhang, K. Lee, Y. Zhang, and H. Lee, "Towards understanding the invertibility of convolutional neural networks," in IJCAI Int. Jt. Conf. Artif. Intell., 2017, pp. 1703-1710.

[47] R. Giryes, G. Sapiro, and A. M. Bronstein, "Deep neural networks with random Gaussian weights: A universal classification strategy?" IEEE Trans. Signal Process., vol. 64, no. 13, pp. 3444-3457, 2016.

[48] X. R. Bao, Y. Pan, C. M. Lee, T. H. Davis, and G. Bao, "Tools for experimental and computational analyses of off-target editing by programmable nucleases," Nat. Protoc., pp. 1-17, 2020.

[49] W. X. Yan, R. Mirzazadeh, S. Garnerone, D. Scott, M. W. Schneider, T. Kallas, J. Custodio, E. Wernersson, Y. Li, L. Gao et al., "BLISS is a versatile and quantitative method for genome-wide profiling of DNA double-strand breaks," Nat. Commun., vol. 8, no. 1, pp. 1-9, 2017. 